\title{
From ultra-dense QCD towards NICA densities: Color-flavor locking and other color superconductors ${ }^{\star}$
}

\author{
Andreas Schmitt ${ }^{\mathrm{a}}$ \\ Mathematical Sciences and STAG Research Centre, University of Southampton, Southampton SO17 1BJ, UK
}

Received: 13 October 2015 / Revised: 26 January 2016

Published online: 16 August 2016

(c) The Author(s) 2016. This article is published with open access at Springerlink.com

Communicated by D. Blaschke

\begin{abstract}
At asymptotically large densities and sufficiently low temperatures, quark matter is a color superconductor in the color-flavor locked phase. I present a brief discussion of this phase and of possible other color superconductors that may appear at densities reached at NICA and in the interior of compact stars.
\end{abstract}

\section{Introduction}

Experiments at NICA are expected to produce the highest baryon densities ever seen in a laboratory, exploring a theoretically extremely challenging and so far poorly understood regime of Quantum Chromodynamics (QCD). One guide for what we may expect and what we should be looking for comes from lower-density physics, for instance from heavy-ion collisions at the Relativistic HeavyIon Collider (RHIC) or the Large Hadron Collider (LHC) as well as from theoretical studies within lattice gauge theory, nuclear physics or various effective and phenomenological approaches. What can we learn from approaching NICA densities "from above", by making use of what we know about even higher densities? From the theoretical point of view, we do have solid knowledge about asymptotically dense QCD, where asymptotic freedom ensures that quarks are weakly interacting. And, extremely dense matter - a few times denser than collisions at NICA will presumably be able to produce - does exist in nature, namely in the interior of compact stars, and astrophysical observations are being used to understand the properties of nuclear and quark matter in a moderately, not asymptotically, dense regime [1]. In the following, I will first comment on the asymptotic regime and then on what we can learn from taking this regime as a starting point for a theoretical journey towards lower densities that can possibly be reached by NICA.

\footnotetext{
* Contribution to the Topical Issue "Exploring strongly interacting matter at high densities - NICA White Paper" edited by David Blaschke et al.

${ }^{a}$ e-mail: a.schmitt@soton.ac.uk
}

\section{Color-flavor locking at ultra-high densities}

In sufficiently cold and dense matter, a condensate of quark Cooper pairs is formed, analogous to Cooper pairing of electrons in a superconductor. Since the interactions are weak at ultra-high density, the ground state can essentially be described within Bardeen-Cooper-Schrieffer (BCS) theory, in particular Cooper pairs are formed only in a small vicinity around the Fermi surface. In order to minimize the free energy, it is advantageous to pair as many quark species as possible. In three-flavor quark matter ${ }^{1}$ there is one unique possibility (up to rotations in color and flavor space that do not change the physics) to pair all quark species, and one can prove rigorously that the resulting state is the ground state at asymptotically large quark chemical potential $\mu$. This state is called the color-flavor locked (CFL) state [2] and is best characterized by its spontaneous symmetry breaking pattern (local groups in square brackets),

$$
\begin{aligned}
& {\left[S U(3)_{c}\right] \times \underbrace{S U(3)_{L} \times S U(3)_{R}}_{\supset\left[U(1)_{Q}\right]} \times U(1)_{B}} \\
& \rightarrow \underbrace{S U(3)_{c+L+R}}_{\supset\left[U(1)_{\tilde{Q}}\right]} \times \mathbb{Z}_{2} .
\end{aligned}
$$

The first line is the symmetry group of QCD, consisting of the color gauge group $S U(3)_{c}$, the chiral symmetry group $S U(3)_{L} \times S U(3)_{R}$ (approximate symmetry, but exact for

\footnotetext{
${ }^{1}$ Even though the three heavy quark flavors will be populated for asymptotically large chemical potentials, they are ignored here because eventually we are interested in much lower densities where only up, down, and strange quarks play a role.
} 
$\mu \rightarrow \infty)$, and the baryon number conservation symmetry $U(1)_{B}$. The CFL state is invariant under transformations of the group in the second line. Several properties of CFL are suggested by this symmetry breaking pattern: CFL is a color superconductor, i.e., the gluons acquire a Meissner mass. A combination of one of the gluons and the photon remains massless, hence there is a residual gauge symmetry group $U(1)_{\tilde{Q}}$ with a "rotated" charge $\tilde{Q}$. Since the "rotated" massless gauge boson is predominantly a photon with only a small admixture of the gluon, CFL is not an electromagnetic superconductor. CFL breaks the chiral symmetry, and as a result there are eight Goldstone modes, very similar to the meson octet at low densities due to the "usual" chiral symmetry breaking. While the "usual" chiral symmetry breaking arises from a condensate of left-handed quarks and right-handed anti-quarks (and vice versa), CFL breaks the chiral symmetry through Cooper pair condensates made of two left-handed or two right-handed quarks, indirectly locked together via color rotations. At asymptotic densities we can neglect all quark masses, and thus the chiral symmetry is exact, leading to exactly massless Goldstone modes. This is no longer true for densities relevant for compact stars or NICA, where the CFL mesons are pseudo-Goldstone modes (if CFL exists at these densities). CFL is a superfluid because baryon number conservation symmetry $U(1)_{B}$ is broken spontaneously. Since this group is an exact symmetry of QCD at all densities, there is another, exactly massless Goldstone mode.

These arguments are rigorous and can be fleshed out and turned into quantitative predictions by first-principle QCD calculations because so far we have chosen to work at sufficiently large densities. However, these densities are larger by many orders of magnitude than any density possibly reached in collider experiments and compact stars. We thus have to ask what happens if we go down in density. Two difficult theoretical challenges arise. First, we leave the region of asymptotic freedom and enter the strong-coupling regime. Currently, there is no reliable first-principle method that tells us how to describe dense QCD matter at large, but not asymptotically large, density, although there are some promising recent attempts to extend lattice calculations into the realm of large chemical potentials $[3,4]$. The second challenge arises from the strange-quark mass $m_{s}$ which can no longer be neglected and thus disrupts the particularly symmetric CFL state. Many studies using perturbative methods, effective theories, and phenomenological models such as the NambuJona-Lasinio (NJL) model have investigated this scenario and the emerging picture is as follows (see ref. [5] for many more details and more references).

\section{Towards NICA densities}

A difference in quark masses induces a difference in Fermi momenta of the quarks that form Cooper pairs, because in the energetically favorable spin-zero channel quarks of different flavor pair. A useful way to think about Cooper pairing of fermions with different Fermi momenta is to first create a fictitious state where both fermion species occupy states up to an average, "common" Fermi surface. This costs free energy. Then, Cooper pairs can form at the common Fermi surface in the usual way, which results in a gain of free energy. If the mismatch in Fermi momenta is small enough, the condensation energy from pairing can overcome the cost and the paired state is favored ${ }^{2}$. This is a very general argument and also applies for instance to cold atomic gases made of two fermion species [7]. In the case of dense quark matter, pairing is favoured if $m_{s}^{2} / \mu$ is small compared to the energy gap $\Delta$ in the quasiparticle excitation spectrum. Starting from CFL and going down in density means to increase $m_{s}^{2} / \mu$, and at large densities we may treat $m_{s}^{2} / \mu$ as a small parameter, going beyond pure CFL in a controlled way. (Of course, $m_{s}, \Delta$, and also the strong-coupling constant are actually functions of $\mu$ and thus in a complete treatment there is only a single parameter, the chemical potential.) At first, CFL reacts to the stress imposed in the strangeness sector by producing anti-strangeness via a kaon condensate $[8,9]$, the kaon being the lightest CFL meson [10]. This modification of the CFL phase is called CFL- $K^{0}$. Importantly, all quarks remain gapped in this phase and thus the low-energy properties of CFL- $K^{0}$ are dominated by the meson sector, including the additional Goldstone mode that arises from the condensation of kaons. (If we take into account the electroweak interaction, which is mandatory for astrophysical applications due to the long time scales involved there, this Goldstone mode acquires a mass because then strangeness conservation, which is spontaneously broken by kaon condensation, is only an approximate symmetry [11].)

All other known color superconductors - with the possible exception of the so-called color-spin locked phase [12, 13] - have unpaired fermionic modes. (These modes may form Cooper pairs among themselves, however with much smaller energy gaps.) The reason is that, if the mismatch in Fermi momenta becomes too large, Cooper pairs start to break, first in certain directions in momentum space. The resulting phases typically have counter-propagating currents. In the simplest case, there is only one current and one counter-propagating current, leading to an anisotropic color superconductor. This is manifest in the so-called curCFL- $K^{0}$ phase, which is the next phase down in density after CFL- $K^{0}[14-16]$. Then, more complicated phases with more supercurrents are formed. They break translational symmetry since they exhibit crystalline structures where the quasiparticle gap varies periodically in position space and vanishes on certain surfaces. These socalled Larkin-Ovchinnikov-Fulde-Ferrell (LOFF) phases

\footnotetext{
2 This picture has an interesting analogue for chiral condensation in the presence of a chemical potential and a background magnetic field [6]: here the chemical potential itself is responsible for a mismatch in energy surfaces, in this case of the fermions and anti-fermions that pair. The analogy to Cooper pairing becomes rigorous because a strong magnetic field, just like the Fermi surface for Cooper pairing, leads to an effective dimensional reduction of the gap equation. Possibly this effect on the chiral condensate is relevant for non-central collisions at NICA, where a strong magnetic field is expected to form.
} 
with CFL pairing pattern have the interesting property of being a superfluid and a crystal at the same time, which makes them highly interesting for explaining the phenomenon of pulsar glitches in certain compact stars [17]. If we further increase $m_{s}^{2} / \mu$, we may reach a regime where only up and down quarks form Cooper pairs $\langle u d\rangle$ ("2SC phase" [18]), or where only single-flavor pairs $\langle u u\rangle,\langle d d\rangle$, $\langle s s\rangle$ are formed, then in the spin-one channel with a multitude of possible phases, not unlike the phases of superfluid ${ }^{3} \mathrm{He}[12,13]$.

Since we do not know the density dependence of the strange-quark mass, we do not know which of these phases resides in which region of the QCD phase diagram. It is possible that the CFL phase persists down to the phase transition to hadronic matter, in which case a smooth crossover to the hadronic phase is conceivable [19-22]. Or there may be a very rich phase structure in between, which would not be surprising given the complicated phase diagrams of ordinary condensed-matter systems. Of course, in this regime close to the hadronic phase we are already deep in the strongly coupled region where we cannot exclude that even more exotic phases occur, such as the quarkyonic phase, which is predicted for QCD if the number of colors $N_{c}$ is large [23] and which may survive in some form for real-world QCD with $N_{c}=3$. At the very least, Cooper pairing will not be restricted anymore to a small vicinity of the Fermi surface, and one can speculate whether QCD exhibits some variant of a BCS-Bose-Einstein-Condensate (BEC) crossover, not unlike ultra-cold atomic gases where the interaction strength can be tuned experimentally [24].

\section{Critical temperature}

For a possible creation of color-superconducting phases at NICA we would like to know at which temperatures they can be expected to be found. In the asymptotic regime, a weak-coupling calculation for CFL yields the following relation between the critical temperature and the zerotemperature quasiparticle gap $\Delta$,

$$
T_{c}=2^{1 / 3} \frac{e^{\gamma}}{\pi} \Delta \simeq 0.71 \Delta,
$$

with the Euler-Mascheroni constant $\gamma$. This relation is similar, but not identical to BCS theory [25]. The difference is the factor $2^{1 / 3}$, which arises due to the two-gap structure of CFL, where 8 quasiparticles have a gap $\Delta$ in their spectrum and one quasiparticle has a gap $2 \Delta$. Such a two-gap structure generically induces a modification in the BCS relation between $T_{c}$ and $\Delta$ (as does an anisotropic condensate). The weak-coupling result for the gap is $\Delta \propto \mu \exp (-$ constant $/ g)$, where $g$ is the strongcoupling constant ${ }^{3}$. The critical temperature acquires corrections from gluon fluctuations, which increase $T_{c}$ by

\footnotetext{
3 Taking into account that due to asymptotic freedom $1 / g^{2}$ increases logarithmically with $\mu$, we know that $\exp$ (-constant/ $g$ ) decreases more slowly than $1 / \mu$. Consequently, $\Delta$ (and thus also $T_{c}$ ) increases with $\mu$ at asymptotically large $\mu$ (while $\Delta / \mu$ decreases).
}

a factor $[1+\mathcal{O}(g)]$ and turn the phase transition from a color-superconducting phase to unpaired quark matter into a first-order phase transition [26]. Extrapolating this result to quark chemical potentials of $\mu \sim 400 \mathrm{MeV}$, assuming $g \sim 3.5$ at that value of $\mu$, gives a critical temperature of about $T_{c} \sim 40 \mathrm{MeV}$, where the enhancement through gluon fluctuations contributes by a factor $\sim 3$. This factor and the result using the BCS gap are bold extrapolations over many orders of magnitude. It is interesting, however, that this estimate is in approximate agreement with estimates from the NJL model which predicts $T_{c} \simeq(10-100) \mathrm{MeV}$ [27-30], or larger if the Polyakov loop is included into the model $[31,32]$. The fact that two completely different approaches agree, one from ultra-high density and one from a phenomenological model with parameters matched to low-density quantities, gives us some confidence that this estimate for the critical temperature is meaningful. One concludes that compact stars (which cool down very quickly from about $10 \mathrm{MeV}$ at their birth to temperatures in the $\mathrm{keV}$ regime) are clearly cold enough to accommodate color superconductors, i.e., if quark matter exists in the core of compact stars it is very likely to be a color superconductor. For matter created at NICA a prediction is less firm, but temperatures around or even below $T_{c}$ are certainly conceivable, especially since all above mentioned corrections to the weak-coupling BCS result tend to enhance the critical temperature.

\section{Phenomenology and observables}

Color-superconducting phases have interesting phenomenological properties which have mostly been studied in view of astrophysical applications. In this context, one is for instance interested in the equation of state, neutrino emissivity, and various transport properties, which can be related to observables such as mass and radius of the star, its cooling behaviour, and its rotation frequency [1]. There is a qualitative difference in the neutrino emissivity and the transport properties of CFL and all other non-CFL color superconductors. The reason is that CFL is the only quark matter phase where all fermionic modes are gapped, and the low-energy properties are entirely determined by the Goldstone modes, see for instance refs. [33-35]. Moreover, since CFL is a superfluid, its hydrodynamic properties are nontrivial because the Cooper pair condensate and the thermal excitations give rise to two fluids, just like the hydrodynamics of superfluid helium is described in a two-fluid picture $[36,37]$. (In the astrophysical context, the normal fluid can sometimes be neglected due to the low temperatures; for heavy-ion collisions at NICA this would presumably be a very bad approximation.) In CFL$K^{0}$ there is even a third fluid component because of the presence of the kaon condensate. The hydrodynamics of high-energy superfluids are studied extensively in the context of compact stars. In particular, hydrodynamic properties of superfluid neutrons and superconducting protons have been investigated, taking into account the formation of vortices and magnetic flux tubes in a rotating star. Besides superfluidity in nuclear matter, it is conceivable 
that a compact star contains superfluid CFL matter in its core. Studies towards a better understanding of relativistic superfluids, starting from a microscopic-field theoretical model, have been put forward recently [38-41], including the discussion of energetic and dynamical instabilities [42] which are of potential relevance for heavy-ion collisions, not unlike the chromo-Weibel instability of the quark-gluon plasma $[43,44]$ that may play a role in the fast thermalization. It will be interesting to see whether NICA may produce sufficiently cold and dense matter to produce superfluidity in quark (or nuclear) matter. This would be particularly intriguing because we know from heavy-ion collisions at RHIC and the LHC that hydrodynamics is a very useful tool to relate microscopic physics (i.e., transport properties such as the shear viscosity) to observables such as the elliptic-flow coefficient $v_{2}$ [45]. Possibly, for matter created at NICA this hydrodynamic description will have to be extended to superfluid hydrodynamics, likely resulting in a qualitative change in $v_{2}$.

Apart from effects on the hydrodynamics, it has also been suggested that the transition to a color-superconducting phase may affect the photon propagator and thus dilepton production in a heavy-ion collision [46-48], although this seems to require the cold quark matter phase to be an electromagnetic superconductor, which is the case for certain single-flavor color superconductors [49], but not for the CFL or 2SC phases. Another interesting possibility is the existence of two critical points at the two ends of a first-order phase transition line, the first of which - at high temperature - is a matter of intense study in the context of heavy-ion collisions since it is expected to have observational signatures in the center-ofmass energy dependence of certain particle number fluctuations $[50,51]$. The existence of a second critical point - at a lower temperature - would have the same observational effects and would indicate a crossover from nuclear matter to the CFL phase at zero temperature [20].

I would like to thank the Science \& Technology Facilities Council (STFC) for support through an Ernest Rutherford Fellowship.

Open Access This is an open access article distributed under the terms of the Creative Commons Attribution License (http://creativecommons.org/licenses/by/4.0), which permits unrestricted use, distribution, and reproduction in any medium, provided the original work is properly cited.

\section{References}

1. A. Schmitt, Lect. Notes Phys. 811, 1 (2010).

2. M.G. Alford, K. Rajagopal, F. Wilczek, Nucl. Phys. B 537, 443 (1999).

3. Michael Fromm, Jens Langelage, Stefano Lottini, Mathias Neuman, Owe Philipsen, Phys. Rev. Lett. 110, 122001 (2013).

4. Gert Aarts, Erhard Seiler, Dénes Sexty, Ion-Olimpiu Stamatescu, Phys. Rev. D 90, 114505 (2014).
5. Mark G. Alford, Andreas Schmitt, Krishna Rajagopal, Thomas Schäfer, Rev. Mod. Phys. 80, 1455 (2008).

6. Florian Preis, Anton Rebhan, Andreas Schmitt, JHEP 03, 033 (2011).

7. M.W. Zwierlein, A. Schirotzek, C.H. Schunck, W. Ketterle, Science 311, 492 (2006).

8. Paulo F. Bedaque, Thomas Schäfer, Nucl. Phys. A 697, $802(2002)$.

9. David B. Kaplan, Sanjay Reddy, Phys. Rev. D 65, 054042 (2002).

10. D.T. Son, Misha A. Stephanov, Phys. Rev. D 61, 074012 (2000).

11. D.T. Son, arXiv:hep-ph/0108260 (2001).

12. Thomas Schäfer, Phys. Rev. D 62, 094007 (2000).

13. Andreas Schmitt, Phys. Rev. D 71, 054016 (2005).

14. Thomas Schäfer, Phys. Rev. Lett. 96, 012305 (2006).

15. Andrei Kryjevski, Phys. Rev. D 77, 014018 (2008).

16. Andreas Schmitt, Nucl. Phys. A 820, 49c (2009).

17. Massimo Mannarelli, Krishna Rajagopal, Rishi Sharma, Phys. Rev. D 76, 074026 (2007).

18. D. Bailin, A. Love, Phys. Rep. 107, 325 (1984).

19. Thomas Schäfer, Frank Wilczek, Phys. Rev. Lett. 82, 3956 (1999).

20. Tetsuo Hatsuda, Motoi Tachibana, Naoki Yamamoto, Gordon Baym, Phys. Rev. Lett. 97, 122001 (2006).

21. Naoki Yamamoto, Motoi Tachibana, Tetsuo Hatsuda, Gordon Baym, Phys. Rev. D 76, 074001 (2007).

22. Andreas Schmitt, Stephan Stetina, Motoi Tachibana, Phys. Rev. D 83, 045008 (2011).

23. Larry McLerran, Robert D. Pisarski, Nucl. Phys. A 796, 83 (2007).

24. Jian Deng, Andreas Schmitt, Qun Wang, Phys. Rev. D 76, 034013 (2007).

25. Andreas Schmitt, Qun Wang, Dirk H. Rischke, Phys. Rev. D 66, 114010 (2002).

26. Ioannis Giannakis, De-fu Hou, Hai-cang Ren, Dirk H. Rischke, Phys. Rev. Lett. 93, 232301 (2004).

27. Harmen J. Warringa, Daniel Boer, Jens O. Andersen, Phys. Rev. D 72, 014015 (2005).

28. Stefan B. Rüster, Verena Werth, Michael Buballa, Igor A. Shovkovy, Dirk H. Rischke, Phys. Rev. D 72, 034004 (2005).

29. D. Blaschke, S. Fredriksson, H. Grigorian, A.M. Oztas, F. Sandin, Phys. Rev. D 72, 065020 (2005).

30. Hiroaki Abuki, Teiji Kunihiro, Nucl. Phys. A 768, 118 (2006).

31. M. Ciminale, G. Nardulli, M. Ruggieri, R. Gatto, Phys. Lett. B 657, 64 (2007).

32. H. Abuki, M. Ciminale, R. Gatto, G. Nardulli, M. Ruggieri, Phys. Rev. D 77, 074018 (2008).

33. Prashanth Jaikumar, Madappa Prakash, Thomas Schäfer, Phys. Rev. D 66, 063003 (2002).

34. Cristina Manuel, Antonio Dobado, Felipe J. LlanesEstrada, JHEP 09, 076 (2005).

35. Mark G. Alford, Matt Braby, Andreas Schmitt, J. Phys. G 35, 115007 (2008).

36. Laszlo Tisza, Nature 141, 913 (1938).

37. L. Landau, Phys. Rev. 60, 356 (1941).

38. Mark G. Alford, S. Kumar Mallavarapu, Andreas Schmitt, Stephan Stetina, Phys. Rev. D 87, 065001 (2013).

39. Mark G. Alford, S. Kumar Mallavarapu, Andreas Schmitt, Stephan Stetina, Phys. Rev. D 89, 085005 (2014).

40. Andreas Schmitt, Phys. Rev. D 89, 065024 (2014). 
41. Andreas Schmitt, Lect. Notes Phys. 888, 1 (2015).

42. Alexander Haber, Andreas Schmitt, Stephan Stetina, Phys. Rev. D 93, 025011 (2016).

43. Ulrich W. Heinz, Nucl. Phys. A 418, 603C (1984).

44. Stanislaw Mrowczynski, Phys. Lett. B 214, 587 (1988) Phys. Lett. B 656, 273(E) (2007).

45. Paul Romatschke, Ulrike Romatschke, Phys. Rev. Lett. 99, 172301 (2007)

46. M. Kitazawa, T. Koide, T. Kunihiro, Y. Nemoto, Phys. Rev. D 65, 091504 (2002)
47. Teiji Kunihiro, Masakiyo Kitazawa, Yukio Nemoto, PoS CPOD07, 041 (2007).

48. D.B. Blaschke, F. Sandin, V.V. Skokov, S. Typel, Acta Phys. Pol. B Suppl. 3, 741 (2010).

49. Andreas Schmitt, Qun Wang, Dirk H. Rischke, Phys. Rev. Lett. 91, 242301 (2003).

50. Misha A. Stephanov, K. Rajagopal, Edward V. Shuryak, Phys. Rev. Lett. 81, 4816 (1998)

51. Christiana Athanasiou, Krishna Rajagopal, Misha Stephanov, Phys. Rev. D 82, 074008 (2010). 